\title{
Detailed and applied anatomy for improved rectal cancer treatment
}

\section{Taxiarchis Konstantinos Nikolouzakis ${ }^{a}$, Theodoros Mariolis-Sapsakos ${ }^{\text {b }}$, Chariklia Triantopoulou ${ }^{c}$, Eelco De Bree ${ }^{d}$, Evaghelos Xynos ${ }^{e}$, Emmanuel Chrysos', John Tsiaoussis ${ }^{\mathrm{a}}$}

Medical School of Heraklion, University of Crete; National and Kapodistrian University of Athens, Agioi Anargyroi General and Oncologic Hospital of Kifisia, Athens; Konstantopouleio General Hospital, Athens; Medical School of Crete University Hospital, Heraklion, Crete; Creta Interclinic, Heraklion, Crete; University Hospital of Heraklion, Crete, Greece

\begin{abstract}
Rectal anatomy is one of the most challenging concepts of visceral anatomy, even though currently there are more than 23,000 papers indexed in PubMed regarding this topic. Nonetheless, even though there is a plethora of information meant to assist clinicians to achieve a better practice, there is no universal understanding of its complexity. This in turn increases the morbidity rates due to iatrogenic causes, as mistakes that could be avoided are repeated. For this reason, this review attempts to gather current knowledge regarding the detailed anatomy of the rectum and to organize and present it in a manner that focuses on its clinical implications, not only for the colorectal surgeon, but most importantly for all colorectal cancer-related specialties.
\end{abstract}

Keywords Anatomy, rectum, cancer, surgery

Ann Gastroenterol 2019; 32 (5): 1-10

\section{Introduction}

Even though rectal anatomy is considered by most clinicians to be a well-known subject, it is still treated as a hot topic, since a search for it across medical databases will retrieve tens of thousands of published works. Interestingly, a recent Canadian study investigated how colorectal cancer (CRC) surgeons define the rectum. The answers were divergent, ranging from a metric definition (e.g., until 12-19 cm from


Heraklion, University of Crete (Taxiarchis Konstantinos Nikolouzakis,


University of Athens, Agioi Anargyroi General and Oncologic Hospital of Kifisia, Athens (Theodoros Mariolis-Sapsakos); 'Radiology Department, Konstantopouleio General Hospital, Athens (Chariklia Triantopoulou); ${ }^{\mathrm{D} D e p a r t m e n t ~ o f ~ S u r g i c a l ~ O n c o l o g y, ~ M e d i c a l ~ S c h o o l ~}$ of Crete University Hospital, Heraklion, Crete (Eelco De Bree); ${ }^{\mathrm{e}}$ Colorectal Surgery, Creta Interclinic, Heraklion, Crete (Evaghelos

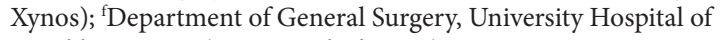
Heraklion, Crete (Emmanuel Chrysos), Greece

\section{Conflict of Interest: None}

Correspondence to: Prof. John Tsiaoussis, Laboratory of AnatomyHistology-Embryology, Medical School of Heraklion, University of Crete, Voutes, 711 10, Heraklion, Crete, Greece,

e-mail: tsiaoussis@uoc.gr

Received 9 April 2019; accepted 25 June 2019; published online 22 July 2019

DOI: https://doi.org/10.20524/aog.2019.0407 the anal verge $[\mathrm{AV}]$ ) to a given landmark (e.g., the part from the sacral promontory) [1]. This study can be considered as indicative of the current overall knowledge on rectal anatomy across CRC-related specialties. On the other hand, even though rectal cancer is a well-studied entity and numerous trials are still running in order to improve the morbidity and mortality rates, there still is a high rate of iatrogenic morbidity $[2,3]$. Therefore, in order to achieve better clinical practice, clinicians first of all have to acquire a thorough understanding of the anatomy and its clinical implications.

\section{The anatomical definition of the rectum: proximal and distal border}

The traditional definition of the rectum relies mostly on its gross anatomy. More specifically, the transition from the sigmoid to the rectum is marked by the coalescence of taeniae coli, the loss of appendices epiploicae and the fusion of the surgical mesocolon. These landmarks are found approximately at the level of the sacral promontory, or $15 \mathrm{~cm}$ from the AV [4-9]. In addition, given the possible clinical benefits, it is helpful to divide the rectum into three parts-upper, middle and lower-measuring the distance from the AV or rarely from the dentate line (DL), using rigid proctosigmoidoscopy or magnetic resonance imaging (MRI). However, age and sex, along with somatometric differences, make the formulation of the rectal borders by metrics or anatomical landmarks rather difficult. Indeed, an MRI based study found that, in the supine position, 
the rectum does not begin at the level of the sacral promontory, but rather at the level of S1-2 [10]. Interestingly, various major trials have used different definitions for the proximal rectal border [11-14], leading to inconsistency in the interpretation of results. Similarly, other surveys have demonstrated considerable variation among surgeons, and other medical practitioners of all colorectal multi-disciplinary team specialties, regarding the rectal borders. Most surgeons (35\%) place the proximal rectal border at the level of the anterior peritoneal reflection, though other anatomical or metric landmarks are also used [1]. In contrast, the various CRC specialties place the proximal border at the "sigmoid take off" on cross-sectional MRI imaging, over all other metrics and anatomical landmarks [15,16] (Fig. 1A). The definition of the distal end of rectum is equally vague. Surgeons locate the anorectal junction at the level where the puborectalis sling surrounds the rectum [4]. In contrast, radiologists use the AV [17] and anatomists the DL as the distal end of the rectum [18]. Although the AV is an external and easily accessible landmark, it is not constant in anatomical terms, as it may vary considerably between individuals depending on body shape and position, and also on the clinician's perception. In contrast, the DL is a well-defined landmark that can provide a more accurate reference point for the measurement of rectal lesions. According to the guidelines of the European Society of Gastrointestinal and Abdominal Radiology, MRI measurements could define the distal end of the rectum as either the anorectal junction or the AV, and this should be stated in the report [19].

\section{Peritoneal covering and anterior peritoneal reflection}

Even though the large bowel is intraperitoneal (appendix, cecum, transverse, and sigmoid colon) or secondary retroperitoneal (ascending and descending colon) the rectum exhibits a unique pattern regarding its peritoneal cover. More specifically, the upper third is usually covered anteriorly and at its sides, the middle only anteriorly, while the lower part is subperitoneal and thus not covered by peritoneum. In this way, a peritoneal fold is formed surrounding the rectum. The deepest and most clinically important part is the anterior peritoneal reflection, or Douglas Pouch. The anterior peritoneal reflection is easily depictable using MRI in $82-90 \%$ of patients [20,21]. Anterior peritoneal reflection is easily identified in the presence of fluid in the pelvic cul-de-sac. In fact, the anterior peritoneal attachment onto the anterior rectal wall exhibits a V-shaped sign on axial MRI section. This low-signal-intensity linear structure forms the so-called "seagull" sign, found at $10.9 \mathrm{~cm}$ from the AV in both sexes [21] (Fig. 1B). However there are cases where the anterior peritoneal reflection is difficult to identify on MRI. These cases are mainly due to poor image quality, motion artifacts, a paucity of pelvic fat planes, retroversion of the uterus, or exophytic rectosigmoid tumors. Nonetheless, the exact position of the anterior peritoneal reflection may vary considerably between individuals. For example, women undergone multiple labors have a considerably deeper cul-de-sac (sometimes even reaching the pelvic floor) (Fig. 1C). This in turn makes the extraperitoneal portion of the rectum shorter than usual $[22,23]$.

\section{Mesorectum and surrounding fascias}

\section{Mesorectum: definition and distribution}

As part of the alimentary tract, the rectum is surrounded by an adipocyte envelope called "meso", in which vessels, nerves and lymph nodes are embedded. This fatty tissue envelope provides a route for all these elements to reach the target organ. Though the distal border of the mesorectum is anatomically obvious, the proximal border is a matter of debate. Some studies considered as proximal border the bifurcation of the superior rectal artery [24], while others consider that the mesorectum extends as far as the proximal border of the rectum, without defining it [25]. From the radiological aspect, the transition from the mesosigmoid to mesorectum is indicated by the "sigmoid take off", and can be identified on cross-sectional sagittal imaging, where the sigmoid colon lies horizontally and the rectum follows the hollow of the sacrum. On this cross-sectional imaging, the superior rectal vessels enter the proximal mesorectum and bifurcate into 2 vessels, adjacent to the inferior portion of the pouch of Douglas approximately at the level of S3 [16]. At this point, the proximal part of the rectum is covered by the mesorectum only laterally and posteriorly, while the middle is encased circumferentially, approximately up to the anterior peritoneal reflection. In the lower part, however, the anterior wall is only rudimentarily covered, as the mesorectum is almost fused with the rectal wall
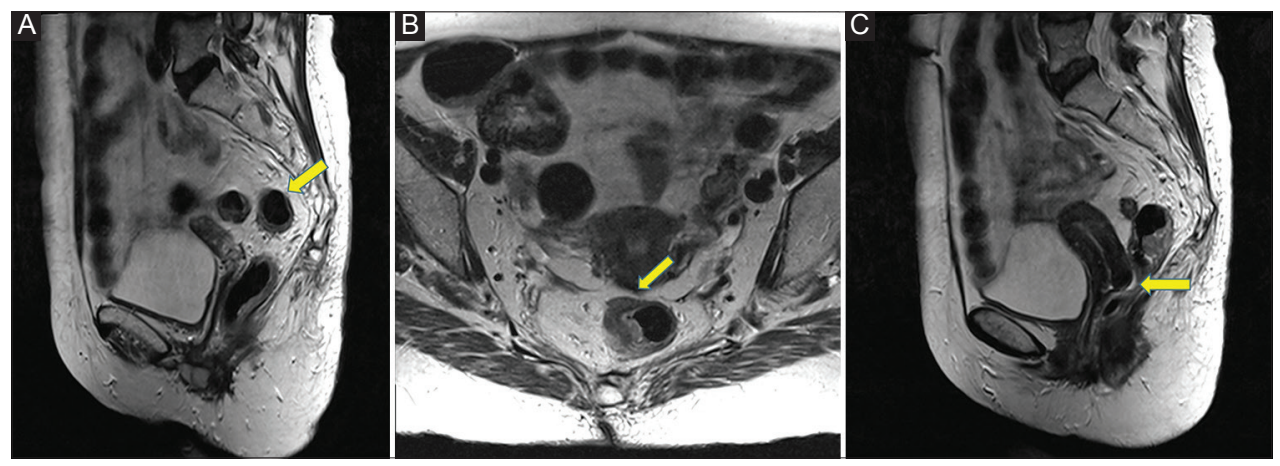

Figure 1 (A) The "sigmoid take off" shown on a sagittal T2-w magnetic resonance (MR) image (arrow). (B) The "seagull" sign on an axial T2-w MR image (arrow). (C) Douglas pouch in a woman, on sagittal T2-w MR image (arrow) 
(Fig. 2A), leaving only the lateral and posterior aspects covered to some extent [24,26-29]. Nonetheless the mesorectal fascia covers the rectum almost universally (except from the lower posterior aspect below the rectosacral fascia, where is difficult to divide it from the rectal wall) [26].

\section{Surrounding fasciae}

\section{Presacral fascia}

At the most posterior aspect lies the presacral fascia, which covers the sacral bone and its venous plexus, extending to the pelvic floor to cover the proctococcygeus muscle, whereupon the level of the levator ani muscle fuses with the mesorectal fascia $[30,31]$.

\section{Parietal pelvic fascia}

Ventrally to the presacral fascia lies the parietal pelvic fascia (Fig. 2B). This dual-lamella structure encases the hypogastric nerves and the pelvic splanchnic nerves, which run through the lateral pelvic walls [32]. However, as it descends towards the levator ani muscle it fuses with the presacral fascia, making it difficult to separate them.

\section{Rectosacral fascia}

On the posteroinferior side of the rectum the rectosacral fascia (Waldeyer's fascia) may be recognized (Fig. 2B). This fascia is described as a thickening of the presacral fascia that descends to meet the mesorectal fascia about 3-5 cm from the anorectal junction [32]. Interestingly, in light of various degrees of endorsement, some anatomists consider the rectosacral fascia to be a form of ligament (rectosacral ligament) [33]. However, the rectosacral fascia exhibits a great variability in its origin and existence. García-Armengol et al reported that 15\% started from the S2 vertebra, 38\% from S3, and 46\% from S4 [31]. Its function appears to be the anchorage of the rectum with the sacral bone, since after its cross-section the motility increases considerably. Between them (the mesorectal fascia and the parietal pelvic fascia) a space is formed, the rectosacral space, separated by the rectosacral fascia into a superior and inferior compartment that communicate with each other [26,34].

\section{Denonvillier's fascia}

The rectoprostatic fascia (Denonvillier's fascia) lies between the anterior side of the rectum and the prostate and seminal vesicles. Its anatomy has been a matter of debate, since some authors maintain that there are no macroscopically distinguishable layers and the so-called posterior layer refers to the mesorectum [35] (Fig. 2A), while others claim not only that there are multiple layers, but also that it continues with the mesorectal fascia [36]. Inside this fascia, nerve fibers from the hypogastric nerve and small vessels leading to the prostate and male genital organs may be recognized. However, sometimes this fascia may not be recognized easily because of the patient's old age [35].

\section{Rectovaginal septum}

The rectovaginal septum consists of 2 thin elements, one anterior and one posterior, clearly distinguishable from one another. More specifically, the posterior petal, which according to some researchers constitutes the mesorectal fascia, originates from the pelvic diaphragm (from the arcus tendineus), ascends always in close contact with the rectum and reaches the peritoneum. Then it is bent backward, passing between the rectum and the peritoneum until it is perfectly fused with the rectum. The anterior petal is fundamentally Denonvillier's fascia, which originates from the pelvic floor and moves upward, adheres to the back of the vagina and continues its cephalic course, at the back of the uterus, to which it adheres [37].

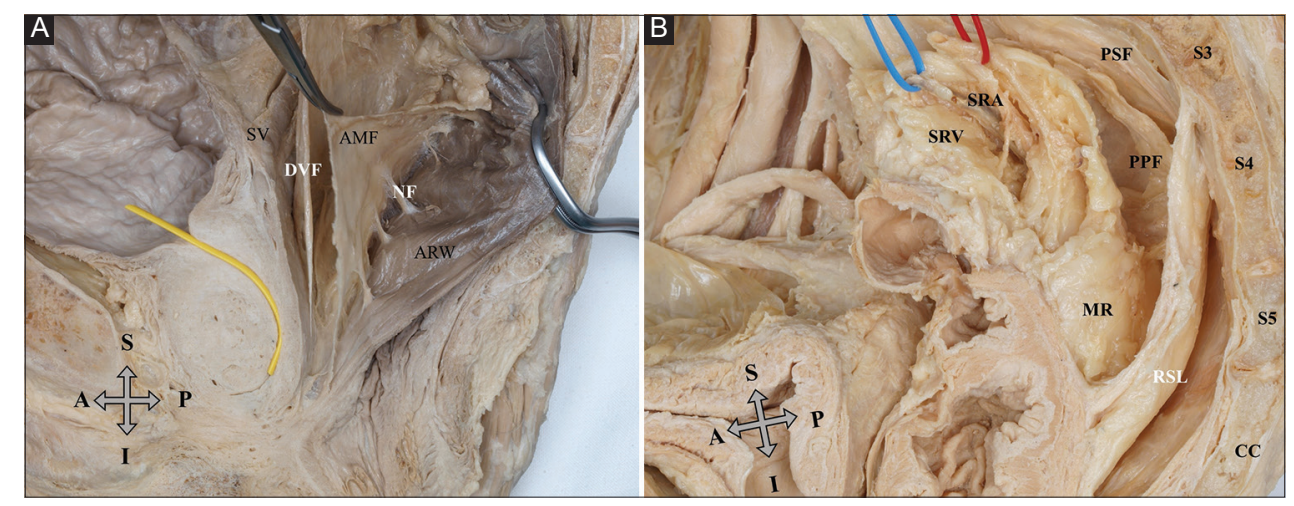

Figure 2 (A) Median sagittal plane in a male cadaveric right hemipelvis. The yellow band indicates the prostatic part of the urethra. AMF, anterior mesorectal fascia; ARW, anterior rectal wall; DVF, Denonvillier's fascia; NF, penetrating rectal nerve fibers; SV seminal vesicles. With permission of Institute of Anatomy, University of Kiel, Germany. (B) Median sagittal plane in a male cadaveric right hemipelvis. Red loop indicates the superior rectal artery (SRA); blue loop indicates the superior rectal vein (SRV). RSL, rectosacral ligament; MR, mesorectum; PSF, presacral fascia; PPF parietal pelvic fascia; S3, S4, S5, sacral vertebrae 3, 4, 5; CC, coccyx. With permission of Institute of Anatomy, University of Kiel, Germany

$S$, superior; A, anterior; $P$, posterior; $I$, inferior 


\section{Lateral rectal ligaments}

Laterally to the rectum we may find the lateral ligaments (Fig. 3A). These are fibrous formations which, depending on the degree of their organization, can take various forms. These structures are a point of intense controversy, as some authors challenge their position, at least as suggested by the various classical surgical handbooks, while others even dispute their existence, since in the absence of discrete strong connective tissue fixing the rectum to the pelvic wall, but rather the presence of loose condensations of the parietal pelvic fascia fusing with the mesorectal fascia, they choose to refer to it as a fascial fusion [38]. Jones et al found that the lateral ligaments of the rectum were present in more than half of the cases (in 15 of the 28 unilaterally and in 3 cases bilaterally). They would form rudimentary fibrous bands from which substantial structures such as vessels and nerves were absent. As for their placement, 17 were located laterally in the rectum, 10 posteriorly and 3 posterolaterally, while their number ranged from 1-5 [39]. However, other research teams managed to find a neurovascular component in $[40,41]$ or above them [42]. Nonetheless, because of the general effort to improve survival rates for patients with rectal cancer (especially those located in the lower rectum), there is a resurgence of research into these ligaments. This can be explained by the fact that, in those cases where the middle rectal artery runs through them, there is a great chance of having accompanying lymphatics towards the lateral pelvic wall, possibly connecting the mesorectal group of lymph nodes with those of the internal iliac artery or the internal obturator artery [43].

\section{Rectal arteries}

The arterial supply of the rectum is traditionally described as being supported by three main vessels: the superior, middle and inferior rectal arteries. However, clinical practice has proved that this description can sometimes be an oversimplification or even misleading.

\section{Superior rectal artery}

The main rectal artery is the superior (SRA), the farthermost branch of the inferior mesenteric artery (IMA). Typically, the IMA is deemed to become the SRA when it crosses the common or internal iliac artery on the left side of the pelvis. Approximately at the level of the S2 or S3 vertebra it divides into a number of small branches that approach the rectum from the posterior aspect [44] (Fig. 2B). In a large study of formalin-fixed cadavers (50 adults and 25 newborns) SRA was found to bifurcate with a large right branch in $81 \%$, to trifurcate in $13 \%$ with branches of the same size, to provide more than 3 branches in $4 \%$, while in $2 \%$ the main trunk of the SRA crossed the posterior side of the rectum in order to create anastomotic loops on both sides. In the majority of cases, the long right branch supplies 2 small vessels that run on the right and anterior sides and lead to a number of smaller ones. The shorter left branch descends undivided on the left side up to a variable point where it branches into smaller vessels [45]. In an angiographic study of 10 rectal specimens, a complex anatomic pattern of small arterial branches and collaterals arising from the axis of the SRA was found, mainly on the lateral and posterior sides. In this case, the mother trunk of the SRA and its daughter vessels (left, right and posterior) gave rise to the small arteries that occurred every $0.5-1 \mathrm{~cm}$ and penetrated through the mesorectum towards the rectal wall in a manner similar to that of the marginal artery and the vasa recta of the colon. Additional (but to a lesser degree) collaterals would rise from the middle and inferior rectal arteries. Interestingly, in the upper and middle rectum, the small arteries had more frequent collaterals than in the lower rectum. However, in the lower rectum, the small arteries were vertically aligned. On the anterior aspect, the homonymous branches of the SRA start from the upper mesorectum and pass anteriorly and inferiorly approximately at the level of the lateral ligaments, where they give collaterals to the middle rectal artery and then penetrate the inferior rectal wall to terminate in the submucosal anal plexus. These anterior branches of the SRA also form variable and tenuous collaterals with the inferior rectal artery and with the posterior branches of the SRA [46].
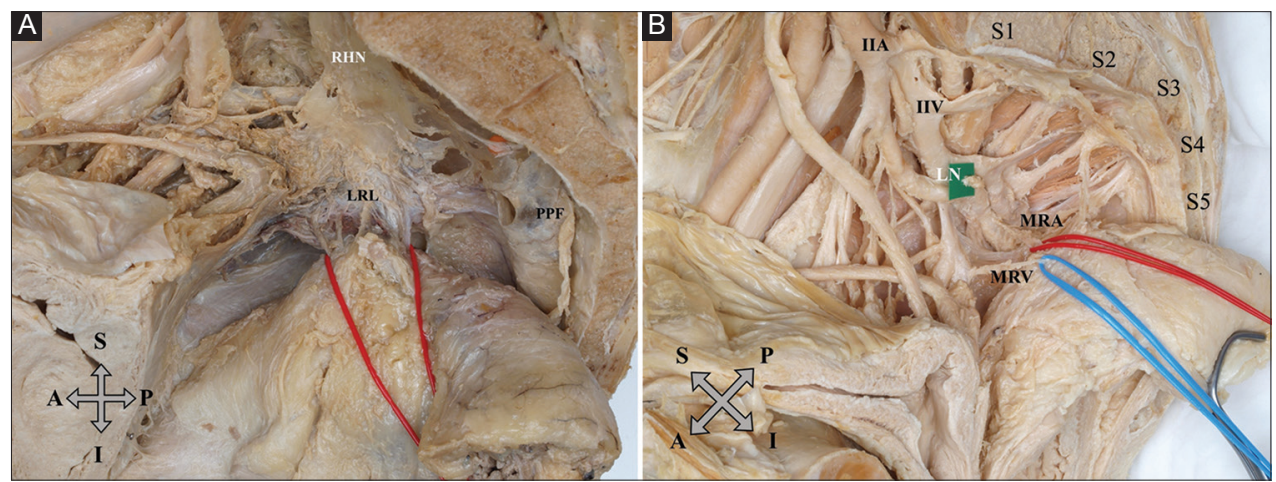

Figure 3 (A) Median sagittal plane in a male cadaveric right hemipelvis. Red loop indicates the right lateral rectal ligament (LRL); RHN indicates the right hypogastric nerve embedded into the parietal pelvic fascia (PPF). With permission of Institute of Anatomy, University of Kiel, Germany. (B) Median sagittal plane in a male cadaveric right hemipelvis. Red loop indicates a middle rectal artery (MRA) arising from the internal iliac artery (IIA); blue loop indicates a middle rectal vein (MRV) draining to the internal iliac vein (IIV); green square indicates an internal iliac lymph node (LN). S1, S2, S3, S4, S5, sacral vertebrae 1, 2, 3, 4, 5. With permission of Institute of Anatomy, University of Kiel, Germany

$S$, superior; A, anterior; P, posterior; I, inferior 


\section{Middle rectal arteries (MRAs)}

MRAs are the most inconsistent as regards their existence (36-57\%) and with respect to their origin and distribution. DiDio et al studied 30 cadavers and found that an MRA was present in only $56.7 \%$ of the cases; in $36.7 \%$ it was bilateral and in $20 \%$ unilateral. As for its origin, the researchers described a cluster of variations. In $40 \%$ the vessel of origin was the internal pudendal artery, in $26.7 \%$ the posterior gluteal artery and in $16.8 \%$ the internal iliac artery, while the other pelvic vessels were less often the site of origin. Moreover, the same investigators found that the most usual level of entrance in the mesorectum was located approximately $6 \mathrm{~cm}$ from the pelvic floor, at the anterior aspect of the rectum (50\%) or the posterior aspect (45\%) [47]. The usual route for the MRA to approach the rectum was provided by the lateral ligaments of the rectum (the rectal stalks) [45] (Fig. 3B).

\section{Inferior rectal arteries (IRAs)}

IRAs are found to be consistent with respect to their existence and their origin from the internal pudendal artery. More specifically, the internal pudendal artery gives rise to the IRA below the levator ani muscle. As it reaches the anorectal junction, the IRA divides into 4 main branches for the distal part of the rectum, the anal canal, and the internal and external anal sphincter, each of which gives rise to 3 more vessels [45]. Being in the rectal wall, it creates anastomotic loops with the MRA (when they exist) or the SRA in the submucosa. However, because of its distribution there is a potentially poorly vascularized area on the posterior lower side of the rectum [48].

\section{Rectal (hemorrhoidal) veins}

The blood is drained from the rectum through its muscularis plexus (the outer plexus), located below the level of the anterior peritoneal reflection until the levator ani muscle, and the submucosal plexus (the inner plexus), which extends from the proximal end of the rectum until the anus. The inner plexus is drained by 2 other plexuses, the superior and the inferior. The superior plexus runs through the rectal columns (and is the one that creates the inner hemorrhoids) and anastomoses with the outer plexus, which finally creates the superior rectal vein, which, with the contribution of the sigmoidal veins, creates the inferior mesenteric vein (part of the portal circulation) (Fig. 2B). The inferior plexus (which gives birth to the outer hemorrhoids) continues towards the anus, where it becomes the inferior rectal vein; this, along with the internal pudendal vein, drains into the internal iliac vein (part of the systematic circulation) [49]. In few cases there is also an accompanying middle rectal vein (MRV). This vein can be recognized in about $32.6 \%$ of cases, in the majority unilaterally. Interestingly the MRV is rarely accompanied by an MRA (6.2\% in one study of 81 hemipelvises). Whatever the case may be, the MRV drain its blood into the internal iliac vein [50].

\section{Rectal lymphatics and lymph nodes: ghost-hunting}

\section{Lymphatic spread}

Since R.J. Heald introduced his holy plane for total mesorectal excision (TME), the surgical community has realized that the majority of rectal lymph nodes are embedded inside the fatty rectal envelope: the mesorectum. However, they are not equally distributed across its volume and do not drain the various rectal parts at random. In fact, it seems that there are certain patterns. Zheng et al, studying 31 patients who underwent TME, tried to unveil the possible pattern of local spread based on the location of the primary lesion. First they classified the tumors as above ( 5 patients) or below (22 patients) the anterior peritoneal reflection. Tumors above the peritoneal reflection appeared to spread across the SRA, with zero metastases along the MRA (when present). Those below the peritoneal reflection appear to be mainly distributed in the SRA and secondarily in the MRA and IRA. Then, the tumors were distinguished according to their position in the rectal surface (anterior, posterior, lateral, circular). It was shown that the preferred metastasis site of the lateral tumors was along a branch of its superior and/or middle rectal artery, with only a small incidence in a branch of its SRA on the opposite side, whereas posterior tumors appeared to be spread along the SRA bilaterally. Thus, most of the lymphatic drainage of the rectum and mesorectum was shown to follow pathways running parallel to the SRA and then the IMA [51]. However, there is a growing body of evidence that those routes of tumor spread are not the only ones. Takahasi et al proved that there is a path connecting the lower rectum with the pelvic sidewalls. It runs laterally and upwards outside the mesorectum to the lateral pelvic walls and internal iliac arteries (mainly though in the internal obturator area) [52]. In fact, tumors located in the lower rectum, and especially those with their lower limit less than $5 \mathrm{~cm}$ from the DL, use this path [53].

\section{Regional lymph nodes}

Several attempts have been made to map the lymph nodes surrounding the rectum, exhibiting a considerable variation in their results, due both to the landmarks used (e.g., peritoneal reflection) and the techniques used to yield the lymph nodes. However, Canessa et al, in a study of 20 cadavers, concluded that along the rectum most lymph nodes are located above the anterior reflection of the peritoneum $(71.4 \%$ were above and $28.6 \%$ were below) [24]. Moreover, most of them appear to be more pronounced in the posterior upper two thirds of the rectum, with only a small number in the lower one third, and even lower in the anterior section along the entire length of the mesorectum $[54,55]$. The size of the lymph nodes, as shown by the Perez et al, appears to be larger in the posterior upper $2 / 3$ than the lateral and lower $1 / 3$ [56]. 


\section{The rectum and its nerve supply}

The innervation pattern of the rectum can be distinguished into two basic interacting systems, one internal and one external. More specifically, the internal system (or intestinal nervous system) consists of a broadly interconnected network formed between 2 plexuses, the myenteric (or Auerbach's plexus) and the submucosal (or Meissner's plexus) [57-59]. The external system is the autonomous nervous system, sympathetic and parasympathetic. Sympathetic preganglionic fibers start from the first three lumbar vertebrae, which, through the white anastomotic branches, enter the sympathetic chain following a downward course and then provide the abdominal visceral nerves that contribute to the pre-aortic plexus. They then continue their course through branches emerging from the pre-aortic plexus, which goes along with the IMA and its branches, and form the inferior mesenteric plexus. Post-ganglionic fibers following the IMA and then the superior rectal artery will innervate the upper rectum $[60,61]$. The lower part of the rectum is innervated by a different path. Nerve fibers starting from the superior hypogastric plexus will enter the pelvis and divide into 2 parts, the 2 hypogastric nerves, consisting of 2 or 3 branches, which follow a parallel course to the ureter approximately $1-2 \mathrm{~cm}$ to the middle line disposed over the lateral sides of the pelvis. Close to its origin, the left hypogastric nerve is often located behind the origin of the SRA. The distal parts of the hypogastric nerves are located about $2-4 \mathrm{~cm}$ below the peritoneum, where they eventually receive sympathetic and parasympathetic sacral fibers from the second, third and fourth lateral roots to form a pelvic plexus (the inferior hypogastric plexus) [62]. At this point, the pelvic splanchnic nerves (nervi erigentes) and sympathetic fibers join the nerve fibers from the hypogastric nerves and radiate towards the middle and lower rectum, forming a "T-junction" that lies close to the posterolateral aspect of the rectum near the pelvic wall $[63,64]$. From this point, the fibers separate into those that innervate the upper rectum, the sigmoid colon, the descending colon and the distal third of the transverse colon, and those that target the middle and lower rectum. The former follow 2 main paths, an ascending path through the inferior and superior hypogastric plexus, to meet the inferior mesenteric plexus, and a retroperitoneal path from which they reach their target organs $[65,66]$; the latter follow the descending course of the pelvic plexuses, running first close to the ureters and the urinary bladder, innervating them, and then turning downwards at the posterior side of the seminal vesicles and the prostate to reach the lower rectum $[60,61]$. A cadaveric dissection of these structures is presented in Fig. 4. According to Gray, parasympathetic fibers from pelvic splanchnic nerves ascend through the superior hypogastric plexus to accompany the inferior mesenteric artery to the distal transverse, descending and sigmoid colon, providing parasympathetic innervation to the left colon, since vagal innervation terminates at the proximal two thirds of the transverse colon. In addition, rami of the pelvic splanchnic nerves ascend on the posterior abdominal wall and distribute directly to the splenic flexure and descending colon [67].

\section{Clinical implications}

\section{Proximal rectal border}

The establishment of an accurate estimation of the proximal border of the rectum is a matter of great importance. This is because, when the sacral promontory is used instead of the sigmoid take-off, a cranial overextension of the proximal rectal border towards the sigmoid is inevitable. Conceivably, this may lead to unnecessary radiation of the sigmoid colon as a result of an extended neo-adjuvant radiation field. Additionally, in cases of sigmoid tumors (especially those at or close to the rectosigmoid junction) there is a greater chance of considering them as rectal ones and treating them accordingly. In this case, there is the risk of exposing the patient to an incorrect treatment [68].

\section{Anterior peritoneal reflection}

The relationship between tumor location and the anterior peritoneal reflection is important in local staging, since rectal tumors invading the anterior peritoneal reflection are staged as T4a lesions and this might change the treatment plan. Unfortunately, prospective studies aiming to validate any correlation between tumor location at the anterior peritoneal reflection with the type of treatment and oncological outcomes are lacking. Anterior rectal tumors located at or above the anterior peritoneal reflection and invading the peritoneum

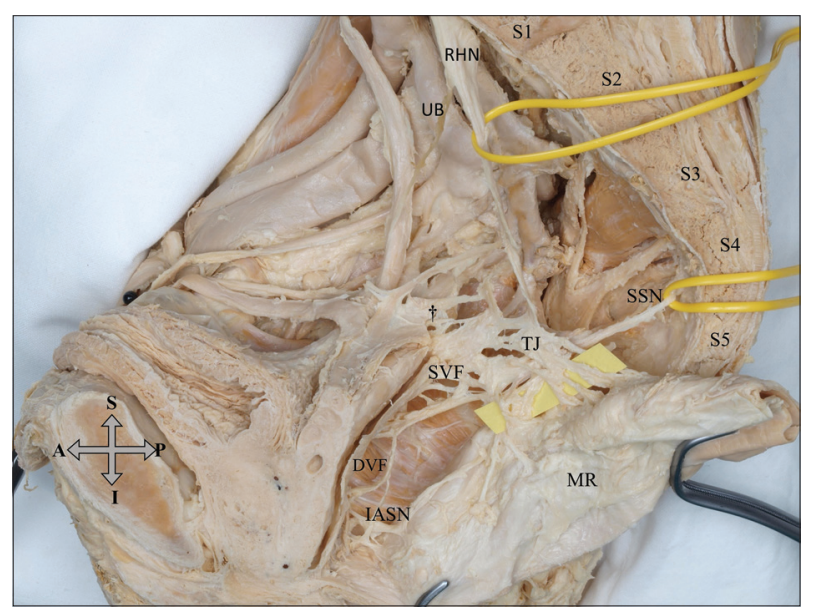

Figure 4 Median sagittal plane in a male cadaveric right hemipelvis. The upper yellow loop indicates the right hypogastric nerve (RHN); UB indicates the branch from the hypogastric nerve for the ureter; the lower yellow loop indicates the sacral (splanchnic) nerves (SSN); the yellow strip indicates the T-junction (TJ); SVF indicates the nerve fibers from the inferior hypogastric plexus radiating towards the seminal vesicles; IASN indicates the nerve fibers for the internal anal sphincter; dagger $(\dagger)$ indicates the hypogastric nerve fibers for the vas deferens, the seminal vesicles and the urinary bladder. DVF, Denonvillier's fascia; MR mesorectum; S1, S2, S3, S4, S5, sacral vertebrae $1,2,3,4,5$. With permission of Institute of Anatomy, University of Kiel, Germany.

$S$, superior; A, anterior; $P$, posterior; I, inferior 
are at great risk of intraperitoneal spread, thus exhibiting a worse prognosis, as compared to those without peritoneal involvement [69]. Moreover, it has been speculated that locally advanced rectal cancers located at or above the anterior peritoneal reflection are not reliably targeted with radiation, since they are more mobile than sub-peritoneal tumors. Thus, inadvertent radiation of small bowel loops lying in the Douglas pouch may take place, resulting in increased morbidity (radiation enteritis) [70]. This is more likely to occur in multiparous older females with a deep Douglas pouch [71]. Conversely, tumors of the middle and lower rectum may be undertreated if considered as being over the anterior peritoneal reflection. In these cases they may not receive neoadjuvant chemoradiotherapy when it is actually indicated [72]. For this reason, it has been proposed that the anterior peritoneal reflection should be the landmark for the selection of the upper boundary of the radiation field $[23,73,74]$. The aforementioned reasons may justify the reluctance of clinicians to irradiate the upper rectum [75,76]. The MERCURY experience highlights the fact that involvement of the anterior peritoneal reflection or the serosa of the intraperitoneal rectum by the tumor on MRI imaging is reported as T4a. If that is resected, it would provide a negative circumferential resection margin [18].

\section{Mesorectum distribution}

The difference in the distribution of the mesorectal volume implies modifications in the treatment of rectal cancer as it may influence the T-stage, upgrading it from a T3 to a T4 [77]. For instance, in case of a locally advanced cancer located anteriorly in the lower rectum, where mesorectal fat cover is thin, local infiltration into neighboring viscera is more likely than in the case of a lateral or posterior rectal tumor, where the covering mesorectum is thicker [69].

\section{Surrounding fasciae}

As the surgeon dissects posteriorly at the level of the "holy plane", separation of the mesorectum from the parietal fascia must be done with caution, in order not to violate the mesorectal fascia and predispose to tumor local recurrence. The key to maintain both compartments intact and to avoid any unnecessary bleeding is to dissect on the avascular "yellow side of the white". For this step, any violation of the parietal pelvic fascia may result in injury of the presacral venous plexus. Moreover, the recognition and dissection of the rectosacral fascia close to its anchoring on the rectal wall increases the mobility of the distal rectum and ensures dissection along the correct plane. In fact, this is of great importance for transanal TME, where the anatomical landmarks are widely different from those identified by the abdominal approach. In addition, when dissecting close to or at Denonviller's fascia or the rectogenital septum, neurovascular involvement is almost inevitable, since small nerve fibers and vessels are sheathed in it, resulting in some degree of urogenital dysfunction.

\section{Arterial supply}

During any low colorectal anastomosis, dissection of the SRA and MRA results in the loss of middle and upper mesorectal collaterals. This has two main side effects: first, the arterial supply of the anastomosis relies on a weak network of small collaterals found close to the rectal wall; and second, increased local intraluminal pressure results in a further decrease of the arterial blood supply, which in turn increases the risk of anastomotic leak [46].

\section{Venous drainage}

Depending on the location of a lesion, different venous networks may carry tumor cells. For the middle and upper rectum, blood is drained through the superior rectal vein, leading it to the portal system via the inferior mesenteric vein. In contrast, the lower and the middle rectum drain mainly through the inferior rectal veins to the systemic circulation via the internal iliac artery. This can explain why tumors of the upper and middle rectum are most often metastasized to the liver, whereas tumors of the lower middle tend to metastasize primarily to the lungs.

\section{Lymph node distribution}

Since the establishment of TME as the standard of care for rectal cancer management, local recurrence rates have dramatically decreased. However, positive nodal disease, and in particular the percentage of positive nodes over the total yielded nodes, carries significant prognostic value [78]. In order for all lymph nodes to be retrieved, the pathologist has to be aware of the distribution of the mesorectal lymph nodes. As shown by Perez et al, mesorectal lymph nodes tend to be found mainly on the posterior side of the mesorectum, and especially in its cranial portion $[53,56]$.

\section{Nerve injury}

The most frequent areas for neural injury while performing a TME are the following:

a) The inferior mesenteric plexus at the origin of the IMA. A $1-1.5 \mathrm{~cm}$ arterial stump is adequate in order to avoid the aforementioned injury [79].

b) The superior hypogastric plexus at the level of the sacral promontory while entering the retrorectal plane [80]. An injury at this point may cause symptoms of bowel dysfunction (ranging from constipation and intermittent defecation with tenesmus to incontinence) [64].

c) The dissection of the retrorectal space may injure the hypogastric nerves, resulting in a certain degree of anorectal incontinence and urogenital dysfunction [81]. Moreover, such an injury may affect the internal anal sphincter nerve, again resulting in anorectal dysfunction. For this 
Table 1 Brief presentation of the most important landmarks in rectal cancer management and their clinical implications and manifestations

\begin{tabular}{|c|c|c|}
\hline Anatomic structure & Clinical significance and manifestations & Reference \\
\hline Sigmoid take-off & $\begin{array}{l}\text { More accurate estimation of the proximal border of the } \\
\text { rectum }\end{array}$ & D’Souza et al., 2018 [16] \\
\hline Anterior peritoneal reflection & Estimation of the risk for intraperitoneal spread & Shepherd et al., 1995 [69] \\
\hline Anteroinferior mesorectum & $\begin{array}{l}\text { Estimation of the risk for urogenital compartment } \\
\text { involvement }\end{array}$ & Shepherd et al., 1995 (59) \\
\hline Presacral fascia & Bleeding of presacral venous plexus if ruptured & Weaver et al., 2015 [84] \\
\hline Rectosacral fascia & $\begin{array}{l}\text { In TME/TaTME dissection close to the rectum will } \\
\text { mobilize the rectum and prevent presacral bleeding }\end{array}$ & Penna et al., 2017 [85] \\
\hline Denonvillier's fascia/rectogenital septum & $\begin{array}{l}\text { Barrier for anterior compartment infiltration and } \\
\text { urogenital dysfunction if dissected }\end{array}$ & $\begin{array}{l}\text { Lindsey et al., } 2000 \text { [35], } \\
\text { Dariane et al., } 2006 \text { [37] }\end{array}$ \\
\hline Posterior collaterals of SRA/MRA & Danger for anastomotic leak if dissected & Allison et al., 2010 [46] \\
\hline Superoposterior mesorectum & The majority of the lymph nodes lie here & $\begin{array}{l}\text { Canessa et al., 2004[53] } \\
\text { Perez et al., } 2008[56]\end{array}$ \\
\hline IMA origin & $\begin{array}{l}\text { Inferior mesenteric plexus injury may cause bowel } \\
\text { dysfunction }\end{array}$ & Liang et al., 2008 [79] \\
\hline Sacral promontory & $\begin{array}{l}\text { Superior hypogastric plexus injury may cause } \\
\text { rectosigmoidal and urogenital dysfunction }\end{array}$ & Liang et al., 2007 [80] \\
\hline Retrorectal space & $\begin{array}{l}\text { Hypogastric nerves injury will cause anorectal } \\
\text { incontinence }\end{array}$ & Wallner et al., 2008 [81] \\
\hline Parietal pelvic fascia & $\begin{array}{l}\text { Inferior hypogastric plexus, internal anal sphincter nerve } \\
\text { injury will cause anorectal and urogenital dysfunction }\end{array}$ & $\begin{array}{l}\text { Keane et al., 2017[82] } \\
\text { Stelzner et al., 2011, } 2018[32,63]\end{array}$ \\
\hline
\end{tabular}

reason, special care should be taken both at the anteriorinferior aspect of the rectum, where nerve fibers for the internal anal sphincter from the pelvic plexus run along the neurovascular bundle, and at the anterior-lateral aspect, where nerve fibers innervate internal anal sphincter [63].

d) At the level of the lateral rectal ligaments, the inferior hypogastric plexus may be injured. Injury to parasympathetic innervation during low anterior resection might result in defecation disorders, a syndrome known as "low anterior resection syndrome" [82]. This can be prevented if dissection of the lateral aspects of the rectum takes place close to lateral mesorectal wall [81].

e) Dissecting at the level of Denonvillier's fascia may jeopardize the integrity of nerves radiating from the hypogastric nerves to the urogenital area [83].

\section{Concluding remarks}

A successful surgical approach in rectal cancer requires an in-depth knowledge of the pelvic anatomy (Table 1 ) $[84,85]$. A universal definition of rectal anatomy in everyday clinical practice is the cornerstone of this foundation. In this vein, the proximal border of the rectum should be defined by the sigmoid takeoff. Moreover, the understanding that the rectum is a dynamic structure that alters its morphology and its coverings as a person gets older may help towards the application of personalized treatment. In this way, clinicians will be able to treat the anterior peritoneal reflection, not as a landmark indicating the middle of the rectum, but rather as a landmark of prognostic significance. An appreciation of the anatomy of the neurovascular supply and lymphatic drainage of the rectum will lower the risk of low anterior syndrome and improve patients' quality of life after rectal cancer surgery.

\section{Acknowledgments}

The MRI images were kindly offered by $\mathrm{Dr}$ C. Triantopoulou. Cadaver dissections were performed at the Anatomy Institute of the Christian Albrechts University of Kiel, by Dr. T. K. Nikolouzakis and Professor Dr. Med. T. Wedel. The authors would like to thank Professor Dr. T. Wedel for providing the pictures and they allow the future use of these pictures for publishing purposes by him.

\section{References}

1. McMullen TP, Easson AM, Cohen Z, Swallow CJ. The investigation of primary rectal cancer by surgeons: current pattern of practice. Can J Surg 2005;48:19-26.

2. Kin C, Snyder K, Kiran RP, Remzi FH, Vogel JD. Accidental puncture or laceration in colorectal surgery: a quality indicator or a complexity measure? Dis Colon Rectum 2013;56:219-225.

3. Octavian Neagoe C, Mazilu O. Pelvic intraoperative iatrogenic 
oncosurgical injuries: single-center experience. $J$ BUON 2016;21:498-504.

4. Jorge JM, Wexner SD. Anatomy and physiology of the rectum and anus. Eur J Surg 1997;163:723-731.

5. Salerno G, Sinnatamby C, Branagan G, Daniels IR, Heald RJ, Moran BJ. Defining the rectum: surgically, radiologically and anatomically. Colorectal Dis 2006;8(Suppl 3):5-9.

6. Heald RJ, Moran BJ. Embryology and anatomy of the rectum. Semin Surg Oncol 1998;15:66-71.

7. Greenstein A. Principles and practice of surgery for the colon, rectum and anus. Gastroenterology 1999;116:1502.

8. Townsend C, Beauchamp RD, Evers BM, Mattox K. Sabiston textbook of surgery: the biological basis of modern surgical practice. $20^{\text {th }}$ Edn, Elsevier, 2016.

9. Gołębiewski A, Murawski M, Losin M, et al. Laparoscopic surgical technique to facilitate management of high anorectal malformations - report of seven cases. Wideochir Inne Tech Maloinwazyjne 2011;6:150-154.

10. Torkzad MR, Blomqvist L. The mesorectum: morphometric assessment with magnetic resonance imaging. Eur Radiol 2005;15:1184-1191.

11. Kusters M, Marijnen CA, van de Velde CJ, et al. Patterns of local recurrence in rectal cancer; a study of the Dutch TME trial. Eur $J$ Surg Oncol 2010;36:470-476.

12. Cedermark B, Dahlberg M, Glimelius B, Påhlman L, Rutqvist LE, Wilking N; Swedish Rectal Cancer Trial. Improved survival with preoperative radiotherapy in resectable rectal cancer. $N$ Engl J Med 1997;336:980-987.

13. Sauer R, Liersch T, Merkel S, et al. Preoperative versus postoperative chemoradiotherapy for locally advanced rectal cancer: results of the German CAO/ARO/AIO-94 randomized phase III trial after a median follow-up of 11 years. J Clin Oncol 2012;30:1926-1933.

14. Dujovny N, Quiros RM, Saclarides TJ. Anorectal anatomy and embryology. Surg Oncol Clin N Am 2004;13:277-293.

15. D'Souza N, de Neree Tot Babberich MPM, Lord A, et al. The rectosigmoid problem. Surg Oncol 2018;27:521-525.

16. D'Souza N, Balyasnikova S, Tudyka V, et al. Variation in landmarks for the rectum: an MRI study. Colorectal Dis 2018;20:O304-O309.

17. Chen Y, Yang X, Wen Z, et al. Fat-suppressed gadolinium-enhanced isotropic high-resolution 3D-GRE-T1WI for predicting small node metastases in patients with rectal cancer. Cancer Imaging 2018;18:21.

18. Salerno G, Sinnatamby C, Branagan G, Daniels IR, Heald RJ, Moran BJ. Defining the rectum: surgically, radiologically and anatomically. Colorectal Dis 2006;8(Suppl 3):5-9.

19. Beets-Tan RGH, Lambregts DMJ, Maas M, et al. Magnetic resonance imaging for clinical management of rectal cancer: updated recommendations from the 2016 European Society of Gastrointestinal and Abdominal Radiology (ESGAR) consensus meeting. Eur Radiol 2018;28:1465-1475.

20. Jung EJ, Ryu CG, Kim G, et al. Is rectal MRI beneficial for determining the location of rectal cancer with respect to the peritoneal reflection? Radiol Oncol 2012;46:296-301.

21. Gollub MJ, Maas M, Weiser M, et al. Recognition of the anterior peritoneal reflection at rectal MRI. Am J Roentgenol 2013;200:97-101.

22. Yiqun S, Tong T, Fangqi L, et al. Recognition of anterior peritoneal reflections and their relationship with rectal tumors using rectal magnetic resonance imaging. Medicine (Baltimore) 2016;95:e2889.

23. Najarian MM, Belzer GE, Cogbill TH, Mathiason MA. Determination of the peritoneal reflection using intraoperative proctoscopy. Dis Colon Rectum 2004;47:2080-2085.

24. Canessa CE, Badía F, Fierro S, Fiol V, Háyek G. Anatomic study of the lymph nodes of the mesorectum. Dis Colon Rectum 2001;44:1333-1336.
25. Lowry AC, Simmang CL, Boulos P, et al; Coloproctology Surgical Society of Australia. Consensus statement of definitions for anorectal physiology and rectal cancer. Colorectal Dis 2001;3:272-275.

26. Chen N, Min PQ, Liu ZY, Wu B, Yang KQ, Lu CY. Radiologic and anatomic study of the extraperitoneal space associated with the rectum. AJR Am J Roentgenol 2010;194:642-652.

27. Kinugasa Y, Murakami G, Uchimoto K, Takenaka A, Yajima T, Sugihara K. Operating behind Denonvilliers' fascia for reliable preservation of urogenital autonomic nerves in total mesorectal excision: a histologic study using cadaveric specimens, including a surgical experiment using fresh cadaveric models. Dis Colon Rectum 2006;49:1024-1032.

28. Lindsey I, Warren BF, Mortensen NJ. Denonvilliers' fascia lies anterior to the fascia propria and rectal dissection plane in total mesorectal excision. Dis Colon Rectum 2005;48:37-42.

29. Lou Z, Zhang W, Meng RG, Fu CG. Massive presacral bleeding during rectal surgery: from anatomy to clinical practice. World $J$ Gastroenterol 2013;19:4039-4044.

30. Muntean V. The surgical anatomy of the fasciae and the fascial spaces related to the rectum. Surg Radiol Anat 1999;21:319-324.

31. García-Armengol J, García-Botello S, Martinez-Soriano F, Roig JV, Lledó S. Review of the anatomic concepts in relation to the retrorectal space and endopelvic fascia: Waldeyer's fascia and the rectosacral fascia. Colorectal Dis 2008;10:298-302.

32. Stelzner S, Holm T, Moran BJ, et al. Deep pelvic anatomy revisited for a description of crucial steps in extralevator abdominoperineal excision for rectal cancer. Dis Colon Rectum 2011;54:947-957.

33. Soreide O, Norstein J. Rectal cancer surgery: optimisation standardisation - documentation. Springer-Verlag Berlin Heidelberg, 1997.

34. Brown G, Kirkham A, Williams GT, et al. High-resolution MRI of the anatomy important in total mesorectal excision of the rectum. AJR Am J Roentgenol 2004;182:431-439.

35. Lindsey I, Guy RJ, Warren BF, Mortensen NJ. Anatomy of Denonvilliers' fascia and pelvic nerves, impotence, and implications for the colorectal surgeon. Br J Surg 2000;87:1288-1299.

36. Kraima AC, West NP, Treanor D, et al. Whole mount microscopic sections reveal that Denonvilliers' fascia is one entity and adherent to the mesorectal fascia; implications for the anterior plane in total mesorectal excision? Eur J Surg Oncol 2015;41:738-745.

37. Dariane C, Moszkowicz D, Peschaud F. Concepts of the rectovaginal septum: implications for function and surgery. Int Urogynecol $\mathrm{J}$ 2016;27:839-848.

38. Ishii M, Shimizu A, Lefor AK, Kokado Y, Nishigori H, Noda Y. Reappraisal of the lateral rectal ligament: an anatomical study of total mesorectal excision with autonomic nerve preservation. Int $J$ Colorectal Dis 2018;33:763-769.

39. Jones OM, Smeulders N, Wiseman O, Miller R. Lateral ligaments of the rectum: an anatomical study. Br J Surg 1999;86:487-489.

40. Pak-art R, Tansatit T, Mingmalairaks C, Pattana-arun J, Tansatit M, Vajrabukka T. The location and contents of the lateral ligaments of the rectum: a study in human soft cadavers. Dis Colon Rectum 2005;48:1941-1944.

41. Nano M, Dal Corso HM, Lanfranco G, Ferronato M, Hornung JP. Contribution to the surgical anatomy of the ligaments of the rectum. Dis Colon Rectum 2000;43:1592-1597.

42. Wang GJ, Gao CF, Wei D, Wang C, Meng WJ. Anatomy of the lateral ligaments of the rectum: a controversial point of view. World J Gastroenterol 2010;16:5411-5415.

43. Takahashi T, Ueno M, Azekura K, Ohta H. Lateral ligament: Its anatomy and clinical importance. Semin Surg Oncol 2000;19:386-395.

44. Sakorafas GH, Zouros E, Peros G. Applied vascular anatomy of the colon and rectum: clinical implications for the surgical oncologist. Surg Oncol 2006;15:243-255. 
45. Michels NA, Siddharth P, Kornblith PL, Parke WW. The variant blood supply to the descending colon, rectosigmoid and rectum based on 400 dissections. Its importance in regional resection: a review of medical literature. Dis Colon Rectum 1965;8:251-278.

46. Allison AS, Bloor C, Faux W, et al. The angiographic anatomy of the small arteries and their collaterals in colorectal resections: some insights into anastomotic perfusion. Ann Surg 2010;251:1092-1097.

47. DiDio LJ, Diaz-Franco C, Schemainda R, Bezerra AJ. Morphology of the middle rectal arteries. A study of 30 cadaveric dissections. Surg Radiol Anat 1986;8:229-236.

48. Vogel P, Klosterhalfen B. [The surgical anatomy of the rectal and anal blood vessels]. Langenbecks Arch Chir 1988;373:264-269.

49. Sharma M, Rai P, Bansal R. EUS-assisted evaluation of rectal varices before banding. Gastroenterol Res Pract 2013;2013:619187.

50. Sato K, Sato T. The vascular and neuronal composition of the lateral ligament of the rectum and the rectosacral fascia. Surg Radiol Anat 1991;13:17-22.

51. Zheng YC, Zhou ZG, Li L, et al. Distribution and patterns of lymph nodes metastases and micrometastases in the mesorectum of rectal cancer. J Surg Oncol 2007;96:213-219.

52. Takahashi T, Ueno M, Azekura K, Ohta H. Lateral node dissection and total mesorectal excision for rectal cancer. Dis Colon Rectum 2000;43:S59-S68.

53. Canessa CE, Miegge LM, Bado J, Silveri C, Labandera D. Anatomic study of lateral pelvic lymph nodes: implications in the treatment of rectal cancer. Dis Colon Rectum 2004;47:297-303.

54. Topor B, Acland R, Kolodko V, Galandiuk S. Mesorectal lymph nodes: their location and distribution within the mesorectum. Dis Colon Rectum 2003;46:779-785.

55. Thakur S, Somashekar U, Chandrakar SK, Sharma D. Anatomic study of distribution, numbers, and size of lymph nodes in mesorectum in Indians: a autopsy study. Int J Surg Pathol 2011;19:315-320.

56. Perez RO, Seid VE, Bresciani EH, et al. Distribution of lymph nodes in the mesorectum: how deep is TME necessary? Tech Coloproctol 2008; 12:39-43.

57. Cossais F, Durand T, Chevalier J, et al. Postnatal development of the myenteric glial network and its modulation by butyrate. Am J Physiol Gastrointest Liver Physiol 2016;310:G941-G951.

58. Cossais F, Leuschner S, Barrenschee M, et al. Persistent increased enteric glial expression of S100 $\beta$ is associated with low-grade inflammation in patients with diverticular disease. J Clin Gastroenterol 2018;53:449-456.

59. Wiskur B, Greenwood-Van Meerveld B. The aging colon: the role of enteric neurodegeneration in constipation. Curr Gastroenterol Rep 2010;12:507-512.

60. Telford ED, Stopford JS. The autonomic nerve supply of the distal colon: an anatomical and clinical study. Br Med J 1934;1:572-574.

61. Woollard HH, Norrish RE. The anatomy of the peripheral sympathetic nervous system. Br J Surg 1933;21:83-103.

62. Church JM, Raudkivi PJ, Hill GL. The surgical anatomy of the rectum - a review with particular relevance to the hazards of rectal mobilisation. Int J Colorectal Dis 1987;2:158-166.

63. Stelzner S, Böttner M, Kupsch J, et al. Internal anal sphincter nerves - a macroanatomical and microscopic description of the extrinsic autonomic nerve supply of the internal anal sphincter. Colorectal Dis 2018;20:O7-O16.

64. Mauroy B, Demondion X, Drizenko A, et al. The inferior hypogastric plexus (pelvic plexus): its importance in neural preservation techniques. Surg Radiol Anat 2003;25:6-15.

65. Gray H, Standring S, Ellis H, Berkovitz BKB. Gray's anatomy: the anatomical basis of clinical practice. $39^{\text {th }}$ edn. Edinburgh: Elsevier Churchill Livingstone, 2004.

66. Lehur PA, Matzel KE, O'Connell PR. Coloproctology. Springer-
Verlag Berlin Heidelberg, 2017.

67. Standring S. Gray's anatomy: the anatomical basis of clinical practice. Elsevier Health Sciences UK, 2008.

68. Paschke S, Jafarov S, Staib L, et al. Are colon and rectal cancer two different tumor entities? A proposal to abandon the term colorectal cancer. Int J Mol Sci 2018;19.

69. Shepherd NA, Baxter KJ, Love SB. Influence of local peritoneal involvement on pelvic recurrence and prognosis in rectal cancer. J Clin Pathol 1995;48:849-855.

70. Birgisson H, Påhlman L, Gunnarsson U, Glimelius B; Swedish Rectal Cancer Trial Group. Adverse effects of preoperative radiation therapy for rectal cancer: long-term follow-up of the Swedish Rectal Cancer Trial. J Clin Oncol 2005;23:8697-8705.

71. Stacey R, Green JT. Radiation-induced small bowel disease: latest developments and clinical guidance. Ther Adv Chronic Dis 2014;5:15-29.

72. Benzoni E, Terrosu G, Bresadola V, et al. Analysis of clinical outcomes and prognostic factors of neoadjuvant chemoradiotherapy combined with surgery: intraperitoneal versus extraperitoneal rectal cancer. Eur J Cancer Care (Engl) 2006;15:286-292.

73. Yun HR, Chun HK, Lee WS, Cho YB, Yun SH, Lee WY. Intraoperative measurement of surgical lengths of the rectum and the peritoneal reflection in Korean. J Korean Med Sci 2008;23:999-1004.

74. Memon S, Keating JP, Cooke HS, Dennett ER. A study into external rectal anatomy: improving patient selection for radiotherapy for rectal cancer. Dis Colon Rectum 2009;52:87-90.

75. Baik SH, Kim NK, Lee YC, et al. Prognostic significance of circumferential resection margin following total mesorectal excision and adjuvant chemoradiotherapy in patients with rectal cancer. Ann Surg Oncol 2007; 14:462-469.

76. Bernstein TE, Endreseth BH, Romundstad P, Wibe A; Norwegian Colorectal Cancer Group. Circumferential resection margin as a prognostic factor in rectal cancer. Br J Surg 2009;96:1348-1357.

77. Popek S, Tsikitis VL, Hazard L, Cohen AM. Preoperative radiation therapy for upper rectal cancer T3,T4/Nx: selectivity essential. Clin Colorectal Cancer 2012;11:88-92.

78. Zuo ZG, Zhang XF, Wang H, et al. Prognostic value of lymph node ratio in locally advanced rectal cancer patients after preoperative chemoradiotherapy followed by total mesorectal excision. Medicine (Baltimore) 2016;95:e2988.

79. Liang JT, Lai HS, Lee PH, Chang KJ. Laparoscopic pelvic autonomic nerve-preserving surgery for sigmoid colon cancer. Ann Surg Oncol 2008;15:1609-1616.

80. Liang JT, Lai HS, Lee PH. Laparoscopic pelvic autonomic nervepreserving surgery for patients with lower rectal cancer after chemoradiation therapy. Ann Surg Oncol 2007;14:1285-1287.

81. Wallner C, Lange MM, Bonsing BA, et al; Cooperative Clinical Investigators of the Dutch Total Mesorectal Excision Trial. Causes of fecal and urinary incontinence after total mesorectal excision for rectal cancer based on cadaveric surgery: a study from the Cooperative Clinical Investigators of the Dutch total mesorectal excision trial. J Clin Oncol 2008;26:4466-4472.

82. Keane C, Wells C, O'Grady G, Bissett IP. Defining low anterior resection syndrome: a systematic review of the literature. Colorectal Dis 2017; 19:713-722.

83. Moszkowicz D, Alsaid B, Bessede T, et al. Where does pelvic nerve injury occur during rectal surgery for cancer? Colorectal Dis 2011;13:1326-1334.

84. Weaver KL, Grimm LM Jr, Fleshman JW Changing the way we manage rectal cancer-standardizing TME from open to robotic (including laparoscopic). Clin Colon Rectal Surg 2015;28:28-37.

85. Penna M, Cunningham C, Hompes R. Transanal total mesorectal excision: why, when, and how. Clin Colon Rectal Surg 2017;30:339-345. 